\title{
Networking, Safety and Security of Underwater Sensor Networks
}

\author{
Dong Yangze, Zhang Gangqiang, He Xudong \\ Science and Technology on Underwater Acoustic \\ Antagonizing Laboratory \\ Shanghai 201108, China
}

\begin{abstract}
In integrated systems for ocean observation, underwater sensing is one of the main tasks. Though there would be various methods to realize the information exchanges, acoustic networking is among the effective ones. Owing to the special application environment, especially the unique communication channel, there would be some different implementation on the networking of sensors. It is concluded into the problem of network protocols: MAC, Routing and Applications. Another important issue is that the safety and security of the network, which would influence its application to a large extent. This paper describes above problems briefly, and presents the solution thoughts in CAMOS.
\end{abstract}

Keywords-underwater sensing, networking protocols, safety and security of underwater acoustic networks

\section{BACKGROUND}

\section{A. CAMOS - Coastal and Arctic Maritime Operations and} Surveillance

The NTNU (Norwegian University of Science and Technology) lighthouse project takes on the ambitious task of developing a robust integrated communication framework that integrates underwater, terrestrial radio and satellite communications in a resilient infrastructure that can support a multitude of applications, primarily within sensor networking. The foreseeable elements of an eventual communication system include a combination of acoustic underwater communications, terrestrial radio and satellite radio [1].

\section{B. Underwater Networking in CAMOS}

As described above, underwater networking will play an important role in CAMOS. It serves as the underwater part of the heterogeneous system, is in charge of the underwater information acquisition and transmission. Based on efficient underwater acoustic communications, the networking would focus on two aspects: one is the construction of the network, including the protocols designation, which will ensure efficient operation; the other is the guarantee of the safety and security of the network, which will ensure normal operation.

\author{
Wang Huakui \\ Science and Technology on Underwater Acoustic \\ Antagonizing Laboratory \\ Zhanjiang 514022, China
}

\section{A. Network Structure}

Compared to other kinds of networks, underwater wireless network has the similar infrastructure. Figure 1 shows the common layer structure [2].

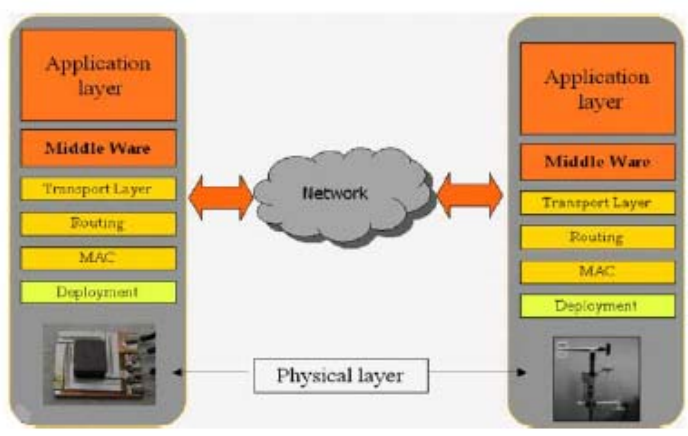

Fig. 1. Infrastructure of underwater wireless network

\section{B. Node Structure}

Concentration to the construction and the performance of the network, we will pay more attention to the MAC (Media Access Control) Layer (Part of Data Link Layer in OSI, what is OSI) and Routing Layer (Part of Network Layer in OSI) in this study. As the physical basis, network nodes will be constructed at first. 2 [3].

The node is with the following structure as shown in Figure

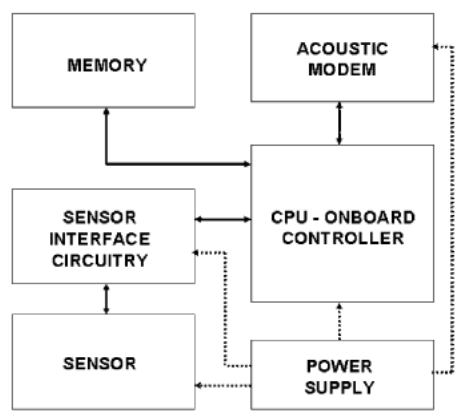

Fig. 2. Node structure of network

\section{UNDERWATER NETWORKING}




\section{Underwater Acoustic Communications}

Though acoustics is the best means for communications in the water, the performance of underwater acoustic communications is limited by the distinct characteristics of sound channel, which lie in the following aspects: slow propagation speed, narrow bandwidth, frequency-selected attenuation, and severe multipath. These may result in low rate, near range, high BER (bit error rate), large time delay, etc.

From 1990's, with the application of coherent processing technique, there has been a shift in high-rate communications. With the rapid development of large scale integrated circuits, OFDM (orthogonal frequency division modulation), MIMO (multi-input multi-output) and TRM (time reversed modulation) techniques are gradually adopted in the study of underwater acoustic communications [4].

\section{Network Protocols[5]}

There are mainly two classes of protocols in networking: MAC protocols and Routing Protocols. The former is a part of Data Link Layer, and the latter belongs to Network Layer.

\section{- MAC Protocols}

Frequency division multiple access (FDMA) is not suitable due to the narrow bandwidth in UWA (underwater acoustic) channels and the vulnerability of limited band systems to fading and multipath.

Time division multiple access (TDMA) shows a limited bandwidth efficiency because of the long time guards required. In fact, long time guards must be designed to account for the large propagation delay and delay variance of the underwater channel, in order to minimize packet collisions from adjacent time slots. Moreover, the variable delay makes it very challenging to realize a precise synchronization, with a common timing reference, which is required for TDMA.

Code division multiple access (CDMA) is quite robust to frequency selective fading caused by underwater multi-paths, since it distinguishes simultaneous signals transmitted by multiple devices by means of pseudo-noise codes that are used for spreading the user signal over the entire available band. This allows exploiting the time diversity in the UWA channel by leveraging Rake filters at the receiver. These filters are designed to match the pulse spreading, the pulse shape and the channel impulse response, so as to compensate for the effect of multi-path. CDMA allows reducing the number of packet retransmissions, which results in decreased battery consumption and increased network throughput.

Carrier sense multiple access (CSMA) prevents collisions with the ongoing transmission at the transmitter side. To prevent collisions at the receiver side, however, it is necessary to add a guard time between transmissions dimensioned according to the maximum propagation delay in the network.

It is noticed that the access schemes described above have been designed for terrestrial sensor networks firstly, whose objective, similarly to underwater wireless sensor networks, is to prevent collisions in the access channel, thus maximizing the network efficiency. These similarities would suggest to tune and apply those efficient schemes in the underwater environment; on the other hand, the main focus in medium access control in terrestrial wireless sensor networks is on energy-latency tradeoffs. Some proposed schemes aim at decreasing the energy consumption by using sleep schedules with virtual clustering. However, these techniques may not be suitable for an environment where dense sensor deployment cannot be assumed. Moreover, the additional challenges due to the underwater channel, such as variable and high propagation delays, and very limited available bandwidth, further complicate the medium access problem in underwater environments.

\section{- Routing Protocols}

During the networking procedure, routing is the peculiar job of network layer in underwater wireless network for multihop strategy is adopted for long distance information conveying.

As mentioned in many literatures, there are many obvious but vast differences between UAN (Underwater Acoustic Network) and other types of networks. Therefore, the existing protocols in WSN (Wireless Sensor Network) and ASN (Ad hoc Sensor Network) may not work directly into UAN without some reformation.

Generally, there are two methods of routing. The first one is virtual circuit routing and the second one is packet-switch routing.

\section{Virtual Circuit Networking Protocols}

In virtual circuit protocols, the networks use virtual circuits to decide on the path at the beginning of the network operation. Virtual-circuit-switch networking protocols can be a better choice for underwater acoustic networks. The reasons lie:

a) They work robust against link failure, which is critical in underwater environment; and

b) They have less signal overhead and low latency, which are needed for underwater acoustic channel environment.

\section{Packet-switch Networking Protocols}

In packet-switch routing, every node that is part of the transmission makes its own routing decision, i.e., decides its next hop to relay the packet. Packet-switch routing can be further classified into proactive routing and reactive routing protocols. Most routing protocols for ground-based wireless networks are packet-switch based.

Proactive routing protocols attempt to minimize the message latency by maintaining up-to-date routing information at all times from each node to any other node. It broadcasts control packets that contain routing table information. Typical protocols include Destination Sequence Distance Vector (DSDV) and Temporally Ordered Routing Algorithm (TORA). However, proactive routing protocols provoke a large signaling overhead to establish routes for the first time and each time the network topology changes. It may not be a good fit in underwater environment due to the high probability of link failure and extremely limited bandwidth there.

In contrast, reactive routing protocols only initiate a route discovery process upon request. Correspondently, each node 
does not need to maintain a sizable "look-up" table for routing. This kind of routing protocols is more suitable for dynamic environment like ad hoc wireless networks. Typical protocol examples are Ad hoc On-demand Distance Vector (AODV), and Dynamic Source Routing (DSR). The shortage of reactive routing protocols is its high latency to establish routing. Similar to its proactive counterpart, flooding of control packets to establish paths is needed, which brings significant signal overhead. The high latency could become much deteriorated in underwater environment because of the much slower propagation speed of acoustic signal compared with the radio wave in the air.

\section{Geographical Protocols}

Besides, there is another type of protocols based on geographical information. With the help of positioning devices, geographical protocols could present promising routing schedules for their scalability feature and limited required signaling.

\section{SAFETY AND SECURITY OF UnDERWATER ACOUSTIC NETWORKS}

The robustness is one of the most important demands of a network. If the network cannot work normally, its functions will be degraded sharply to a collection of individual sensor nodes, which would be no use at all.

From the point of view of the results, it is no differences between the destroying caused by safety and security problems. But thinking of the causations, it is important to discriminate the differences, which might influence the foregoing measures.

As one knows, the application environment may bring tremendous influences to the systems. From the point of view of the robustness of UAN, there are two factors should be taken into considerations.

\section{A. Severe Natural Environment}

Surely, underwater wireless networks are deployed in underwater, especially in the sea. An obvious fact is that most of the practical (experimental) ones are in the littoral sea, which is one of the acknowledged difficult communication channels. In such channels, the successful communication probability would be lowered to some extent. Thus the energy of the power supply (usually batteries) would be consumed rapidly.

Another impact of such environment to underwater wireless network is that the nodes might not be strictly static own to the water float, which would produce troubles for precise localization.

The third circumstance is that the erosion of the sea water to the nodes, which would accelerate their collapse extremely.

\section{B. Hostile Attacks}

Whether or not it is used in the civil fields, hostile attacks are potential threats to UAN for various purposes (e.g., by terrorists).
The attacks may be classified into three categories: attacks to nodes, to communication links, and to the network protocols.

\section{Comments}

In summary, UAN works in a dangerous environment, and it is prone to be destroyed by either natural or man-made breaks.

To the former, it's a safety problem, and the latter is a problem of security. Together with the two assurances, we can say that the UAN is a robust one.

\section{WHAT WILL BE DONE}

In CAMOS, underwater wireless network is an important constitution. Before the information from underwater going out of the water, it is a problem of construction of a robust sensor network.

The robustness of the network itself comes from several aspects: reliable physical layer support, reliable MAC and Routing Protocols, safety and security measures, etc.

In our study, focuses will be on networking topology, networking protocols, safety and security.

\section{A. Network Topology}

Because the information from underwater sensors would be sent out of water, and instructions from above water would sent to relevant network nodes, underwater wireless networks are often with the topology of concentration. The central node is usually called a sink (or gateway) in charge of the information exchange. According to the concrete applications' demands, the topology would be various in order to get optimal properties.

This study will investigate several topologies, and present the comparisons for selection by CAMOS.

Figure 3 presents a typical scenario.

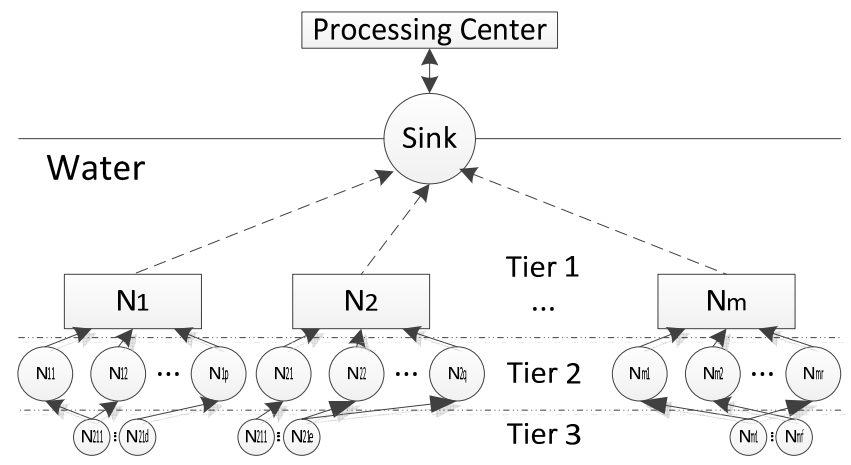

Fig. 3. A Simplified illustration of UAN topology

\section{B. MAC Protocols}

FDMA, TDMA and CDMA are all fixed access methods, while CSMA, MACA, ALOHA, etc. are dynamic access ones.

The main factors should be taken into consideration of MAC protocol design lie in: energy efficiency; extendibility; 
collision avoidance; channel availability; time delay; throughput; justice. Among them, energy efficiency is the most significant one. Therefore, most of the MAC protocols devote into the realization of it.

Generally, there are two main categories of MAC protocols: competitive protocols and assigned protocols. Based on these two classes of protocols, mixed MAC protocols come up thereafter.

This study will analyze some MAC protocols, and present new ones appropriate to CAMOS.

\section{Routing Protocols}

To evaluate a routing protocol, following aspects should be taken into consideration: energy efficient, which will determine the lifecycle of the network. This is the primary goal of protocol design; robustness, which can guarantee the basic functions of the network on condition that some of the nodes goes wrong; extendibility, which makes it easy to cooperate with other nodes; time delay, which should be minimized during the design.

This study will propose two or three routing protocols based on above principles to meet the demands of CAMOS.

\section{Cross-layer Design}

While all the research on underwater networking so far has followed the traditional layered approach for network design, it is an increasingly accepted opinion in the wireless networking community that the improved network efficiency, especially in critical environments, can be obtained with a cross-layer design approach.

This study will consider this viewpoint, trying to do some fusion work about MAC and Routing protocols, as well as the topology design, in order to gain more profits for CAMOS application.

\section{E. Network Safety and Security}

In section 3, we analyzed the environment of underwater wireless networks, which would bring safety and security problems.
There are two measures to guarantee the safety and security of network. One is to add security measures during the design of network, including all steps of the construction and the other is trying to re-organize the partial-destroyed network to acceptable usage.

In this study, the first measure would be reflected in the study of IV.B-IV.D. The second measure would be considered, including the causation analysis, destroying extent analysis, and re-organizing methods.

\section{F. Physical Layer Support}

In this study, at least 4 network nodes with reliable acoustic communications are needed for a sea trial.

\section{SUMMARY}

In this paper, CAMOS is first introduced which is a lighthouse project of NTNU. Within the scope of CAMOS, underwater acoustic networks would play important roles.

After briefly introducing the bases of underwater acoustic networks, some recommendations are put forward for further study of CAMOS.

\section{ACKNOWLEDGMENT}

This work was partly pursued in NTNU when I was a visiting professor there. Thanks will go to NTNU, China Scholarship Council, and Professor Hefeng Dong.

\section{REFERENCES}

[1] Yangze Dong. Study on Construction and Security of Underwater Acoustic Networks. NTNU research report, Trondheim, March 2014.

[2] F. Hu, Neeraj K. Sharma, "Security considerations in ad hoc sensor networks”, Ad Hoc Networks, vol. 3, pp. 69-89, March 2005.

[3] I. F. Akyildiz, et al., "Underwater acoustic sensor networks: research challenges”, Ad Hoc Networks, vol. 3, pp. 257-279, March 2005.

[4] Zaihan Jiang, "Underwater acoustic networks - issues and solutions", International Journal of Intelligent Control and Systems, vol. 13, pp. 152-161, September 2008.

[5] John Cowley, Communications and Networking (second edition). New York: Springer London Heidelberg New York Dordrecht, 2012. 